Reprod. Nutr. Dévelop., 1986, 26 (1 B), 297-298.

\title{
Influence de l'addition de phosphore sur la digestion d'une paille traitée à l'ammoniac par les microorganismes du rumen en fermenteur semi-continu
}

\author{
Michelle DURAND, Ph. BEAUMATIN, Christiane DUMAY, F. MESCHY, Sylvie KOMl- \\ SARCZUK
}

Station de Recherches de Nutrition, I.N.R.A., 78350 Jouy-en-Josas, France.

Summary. The rumen simulation technique was used. Supplementing with $P(120 \mathrm{mg} / \mathrm{l})$ the artificial saliva infusing the fermenters containing $15 \mathrm{~g}$ ammoniated straw per bag improved significantly fiber digestion, VFA and gas production but protein in the effluent was not much affected. The results emphasize the role of $P$ in fiber digestion.

Il a été montré in vitro, à l'aide d'un régime purifié, qu'une subcarence en phosphore $(\mathrm{P})$, modifie l'activité microbienne et réduit la digestion de la cellulose dans le rumen (Durand $e t$ al., 1983). La teneur en $P$ des pailles étant relativement faible (de 0,7 à $1,5 \mathrm{~g} / \mathrm{kg} \mathrm{MS}$ ) nous avons recherché l'effet de l'addition de $P$ sur la digestion microbienne d'une paille traitée à l'ammoniac anhydre en utilisant la technique du fermenteur semi-continu (" Rusitec ») de Czerkawski et Breckenridge (1977).

Matériel et méthodes. L'inoculum (contenu solide et liquide de rumen) provient de 3 moutons qui consomment une paille traitée à l'ammoniac complémentée en minéraux et vitamines. L'équivalent de $15 \mathrm{~g}$ de matière sèche (MS) de la même paille sont introduits dans chaque sachet nylon du fermenteur $(160 \times 80 \mathrm{~mm}$, voie de maille de $150 \mu \mathrm{m})$. Cette paille apporte par sachet $13,7 \mathrm{mg}$ de $P$ et $192 \mathrm{mg}$ d'azote ( $\mathrm{N}$ total) dont $105 \mathrm{mg}$ sont solubles dans un tampon phosphate-bicarbonate. Le plan expérimental comprend 2 périodes consécutives de 10 jours ( 6 jours d'adaptation suivis de 4 jours de mesures) au cours desquelles les tampons sans phosphate $(-\mathrm{P})$ ou supplémentés par $120 \mathrm{mg} P / \mathrm{I}$ sous forme de $\mathrm{Na}_{2} \mathrm{HPO}_{4}(+P)$ et contenant ou non de l'urée $(150 \mathrm{mg} / \mathrm{l})(+\mathrm{U}$ et $-U)$ sont perfusés à raison de un I environ par fermenteur et par $24 \mathrm{~h}$.

Les tampons sont rendus iso-sodiques $(3,65 \mathrm{~g} \mathrm{Na} / 1)$ par addition de $\mathrm{NaHCO}_{3}$. Une expérience préliminaire ayant montré que les variations entre fermenteurs étaient inférieures aux variations journalières dans un même fermenteur, les 4 fermenteurs $(A, B, C, D)$ sont répartis selon le dispositif suivant :
Fermenteurs
$A+B$
$C+D$
Périodes
I: $-P(-U) ; I I:-P(+U)$
$1:+P(-U) ; 11:+P(+U)$

En outre, les fermenteurs $A$ et $B$ ont été supplémentés en $P$ au cours $d^{\prime} u n e$ période supplémentaire (III) afin d'examiner si cette supplémentation permettait de restaurer leur activité. On détermine journellement les volumes de gaz produit, les teneurs des effluents en $\mathrm{N}-\mathrm{NH}_{3}, \mathrm{~N}$-protéique (précipité par l'acide trichloracétique), acides gras volatils (AGV) et la MS des résidus des sachets. La matière organique (MO) et les composés pariétaux (Goering et Van Soest, 1970), sont 
dosés sur les résidus cumulés des sachets de deux jours consécutifs par fermenteur.

Résultats et discussion. Dans les milieux supplémentés en $P$, la perfusion d'urée (période II) améliore significativement tous les paramètres de l'activité fermentaire ainsi que la production d'azote protéique ; par contre, dans les milieux carencés en $P$, I'addition d'urée reste pratiquement sans effet (tabl. 1).

TABL. 1. - Effet de l'addition d'urée et de P sur l'activité microbienne.

\begin{tabular}{cccc}
$\begin{array}{c}\text { Période I } \\
(-U)\end{array}$ & $\begin{array}{c}\text { Période II } \\
(+U)\end{array}$ & $\begin{array}{c}\text { Période III } \\
(+U)\end{array}$ \\
\hline$-P+P$ & $-P$ & $+P$ & $+P$ \\
\hline
\end{tabular}

\begin{tabular}{|c|c|c|c|c|c|}
\hline $\begin{array}{l}\text { Digestion sachets (n }=4 \text { ) } \\
\text { MO \% } \\
\text { Hémicellulose \% } \\
\text { Cellulose \% }\end{array}$ & $\begin{array}{l}23,2^{\mathrm{a}} \\
23,5^{\mathrm{a}} \\
18,1^{\mathrm{a}}\end{array}$ & $\begin{array}{l}28,0^{\mathrm{b}} \\
29,3^{\mathrm{b}} \\
25,8^{\mathrm{b}}\end{array}$ & $\begin{array}{l}23,2^{a} \\
25,0^{a} \\
17,6^{a}\end{array}$ & $\begin{array}{l}35,9^{\mathrm{ca}} \\
37,4^{\mathrm{c}} \\
38,2^{\mathrm{cA}}\end{array}$ & $\begin{array}{l}39,5^{\mathrm{cB}} \\
39,7^{\mathrm{c}} \\
41^{\prime}, 8^{\mathrm{CB}}\end{array}$ \\
\hline Gaz $\mathrm{ml} / 24 \mathrm{~h}(\mathrm{n}=8)$ & $488^{A a}$ & $651^{\mathrm{b}}$ & $430^{a}$ & $870^{\mathrm{Bc}}$ & $1073^{\mathrm{Cc}}$ \\
\hline $\begin{array}{l}\text { Effluents } \\
\qquad \begin{array}{l}\text { AGV mmoles } / 24 \mathrm{~h}(\mathrm{n}=8) \\
\mathrm{N} \text {-protéique } \mathrm{mg} / 24 \mathrm{~h}(\mathrm{n}=8)\end{array}\end{array}$ & $\begin{array}{l}28,3^{A a} \\
50,0^{A}\end{array}$ & $\begin{array}{l}32,1^{\mathrm{Ba}} \\
49,7^{\mathrm{A}}\end{array}$ & $\begin{array}{l}27,4^{\mathrm{Aab}} \\
54,1^{\mathrm{AC}}\end{array}$ & $\begin{array}{l}40,4^{\mathrm{C}} \\
58,2^{\mathrm{BC}}\end{array}$ & $\begin{array}{l}43,2 \mathrm{c} \\
57,9 \mathrm{BC}\end{array}$ \\
\hline
\end{tabular}

$A \neq B \neq C: P<0,05 ; a \neq b \neq c: P<0,01$.

Bien que l'addition de $P$ n'élève pas significativement les valeurs de $\mathrm{N}$-protéique, elle accroît significativement tous les paramètres de fermentation et ce phénomène est nettement amplifié par la perfusion d'urée. Les résultats de la période III montrent que la supplémentation en $\mathrm{P}$ du milieu préalablement carencé a permis de retrouver en totalité, et même parfois de dépasser l'activité microbienne du milieu qui avait été supplémenté en $P$ dès le début de l'incubation (tabl. 1). En outre, l'accroissement de la digestion de la cellulose s'accompagne d'une modification du faciès fermentaire avec élévation du pourcentage molaire d'acétate dans tous les milieux supplémentés en $P(75 \%$ contre $71 \%)$.

L'observation selon laquelle l'apport d'urée n'a pas stimulé la digestion microbienne dans les milieux carencés en $P$, contrairement aux milieux supplémentés, indique que le phosphore est le premier facteur limitant de la digestion des composés pariétaux de cette paille. Nos observations sont en accord avec les résultats de Francis, Gawthorne et Storer (1978) qui montrent l'effet stimulant des ions phosphates sur l'activité des cellulases isolées de contenu de rumen.

En conclusion, cette expérience a permis de mettre en évidence le rôle primordial de $P$ en tant que facteur d'optimisation de la digestion microbienne des fourrages pauvres et notamment de leur fraction cellulosique.

Czerkawski J. W., Breckenridge G., 1977. Design and development of a long-term Rumen Simulation Technique (Rusitec). Br. J. Nutr., 38, 371-384.

Durand M., Boxebeld A., Dumay C., Beaumatin Ph., 1983. Influence of the level of dietary phosphorus on urea utilization by rumen microorganisms in lambs, 263-266. In Metabolisme et nutrition azoté. Coll. I.N.R.A., Vol. II, éd. I.N.R.A. Publ. Versailles.

Francis G. L., Gawthorne J. M., Storer G. B., 1978. Factors affecting the activity of cellulase isolated from the rumen digesta of sheep. Appl. environ. Microbiol., 36, 643-649.

Goering H. K., Van Soest P. J., 1970. Forage fiber analyses. Agriculture Handbook $n^{\circ} 379$ Agric. Res. Serv. U. S. Department of Agriculture. 